\title{
Front Matter: Volume 9742
}

, "Front Matter: Volume 9742," Proc. SPIE 9742, Physics and Simulation of Optoelectronic Devices XXIV, 974201 (12 July 2016); doi:

$10.1117 / 12.2239349$

SPIE. Event: SPIE OPTO, 2016, San Francisco, California, United States 


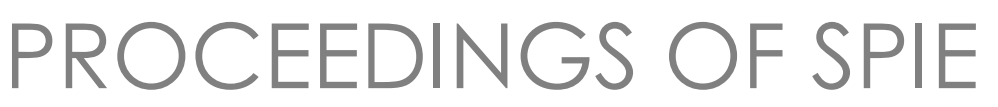

\title{
Physics and Simulation of Optoelectronic Devices XXIV
}

\author{
Bernd Witzigmann \\ Marek Osiński \\ Yasuhiko Arakawa \\ Editors
}

15-18 February 2016

San Francisco, California, United States

Sponsored and Published by

SPIE 
The papers in this volume were part of the technical conference cited on the cover and title page. Papers were selected and subject to review by the editors and conference program committee. Some conference presentations may not be available for publication. Additional papers and presentation recordings may be available online in the SPIE Digital Library at SPIEDigitallibrary.org.

The papers reflect the work and thoughts of the authors and are published herein as submitted. The publisher is not responsible for the validity of the information or for any outcomes resulting from reliance thereon.

Please use the following format to cite material from these proceedings:

Author(s), "Title of Paper," in Physics and Simulation of Optoelectronic Devices XXIV, edited by Bernd Witzigmann, Marek Osiński, Yasuhiko Arakawa, Proceedings of SPIE Vol.9742 (SPIE, Bellingham, WA, 2016) Six-digit Article CID Number.

ISSN: 0277-786X

ISSN: 1996-756X (electronic)

ISBN: 9781628419771

Published by

SPIE

P.O. Box 10, Bellingham, Washington 98227-0010 USA

Telephone +1 3606763290 (Pacific Time) · Fax +1 3606471445

SPIE.org

Copyright (C) 2016, Society of Photo-Optical Instrumentation Engineers.

Copying of material in this book for internal or personal use, or for the internal or personal use of specific clients, beyond the fair use provisions granted by the U.S. Copyright Law is authorized by SPIE subject to payment of copying fees. The Transactional Reporting Service base fee for this volume is $\$ 18.00$ per article (or portion thereof), which should be paid directly to the Copyright Clearance Center (CCC), 222 Rosewood Drive, Danvers, MA 01923. Payment may also be made electronically through CCC Online at copyright.com. Other copying for republication, resale, advertising or promotion, or any form of systematic or multiple reproduction of any material in this book is prohibited except with permission in writing from the publisher. The CCC fee code is $0277-786 \mathrm{X} / 16 / \$ 18.00$.

Printed in the United States of America.

Publication of record for individual papers is online in the SPIE Digital Library.

\section{SPIE. DIGITAL}

Paper Numbering: Proceedings of SPIE follow an e-First publication model. A unique citation identifier (CID) number is assigned to each article at the time of publication. Utilization of CIDs allows articles to be fully citable as soon as they are published online, and connects the same identifier to all online and print versions of the publication. SPIE uses a six-digit CID article numbering system structured as follows:

- The first four digits correspond to the SPIE volume number.

- The last two digits indicate publication order within the volume using a Base 36 numbering system employing both numerals and letters. These two-number sets start with $00,01,02,03,04$, $05,06,07,08,09,0 A, 0 B \ldots$. OZ, followed by 10-1Z, 20-2Z, etc. The CID Number appears on each page of the manuscript. 


\title{
Contents
}

\author{
vii Authors \\ ix Conference Committee
}

SESSION 1 LIGHT EMITTING DIODES

974202 Challenges towards the simulation of GaN-based LEDs beyond the semiclassical framework (Invited Paper) [9742-1]

\section{SESSION 2 PHOTODETECTORS}

974207 A Monte Carlo simulator for noise analysis of avalanche photodiode pixels in low-light image sensing [9742-6]

974208 Modeling and simulation of a 3D-CMOS silicon photodetector for low-intensity light detection [9742-7]

9742 OA Toward designing back-illuminated CMOS image sensor based on 3D modeling [9742-9]

\section{SESSION 3 OPTICAL INJECTION AND FEEDBACK}

9742 OB Dynamics of nanolasers subject to optical injection and optical feedback (Invited Paper) [9742-10]

9742 OC Randomness extraction from a chaotic laser diode with dispersive self-injection (Invited Paper) [9742-11]

9742 OD Lasing state hysteresis in a two-state quantum dot laser via optical injection [9742-12]

9742 OE Gain compression effect on the modulation dynamics of an optically injection-locked semiconductor laser using gain lever [9742-13]

9742 OG Dynamics of optically-injected semiconductor nanolasers [9742-15]

\section{SESSION 4 NONLINEAR LASER DYNAMICS}

$9742 \mathrm{OH} \quad$ Modeling of ultrashort pulse generation in mode-locked VECSELs (Invited Paper) [9742-16]

$974201 \quad$ Dynamic model of pulsed laser generators based on multi-junction N-p-N-i-P heterostructures [9742-17]

$97420 \mathrm{~J}$ Investigation on electro-optic optical comb generation with higher spectral resolution and bandwidth [9742-18] 
9742 OK Coherence properties of fast frequency swept lasers revealed via full electric field reconstruction [9742-19]

$9742 \mathrm{OL} \quad$ Spectral filtering effects in synchronized semiconductor laser networks [9742-20]

SESSION 5 ELECTROMAGNETICS

9742 OM Model order reduction for the time-harmonic Maxwell equation applied to complex nanostructures [9742-21]

$9742 \mathrm{ON} \quad$ Criteria of backscattering in chiral one-way photonic crystals [9742-22]

9742 OP Diffraction patterns from multiple tilted laser apertures: numerical analysis [9742-68]

\section{SESSION 6 QUANTUM DOTS}

9742 OS Study of electro-absorption effects in 1300nm In(Ga)As/GaAs quantum dot materials [9742-27]

SESSION 7 NON-CLASSICAL LIGHT

9742 0X Creation and control of entanglement by time-delayed quantum-coherent feedback [9742-32]

\section{SESSION 8 SILICON OPTO-/ELECTRONICS}

$97420 Z$ Integrated nanophotonic devices for optical interconnections (Invited Paper) [9742-34]

$974211 \quad$ Pushing the limits of silicon transistors [9742-36]

\section{SESSION 9 SEMICONDUCTOR LASERS}

974214 The role of optoelectronic feedback on Franz-Keldysh voltage modulation of transistor lasers [9742-40]

\section{SESSION 10 ACTIVE MATERIALS}

974216 Feedback control of optomechanical systems [9742-43]

974217 Photon momentum and optical forces in cavities [9742-44]

974218 Theoretical investigations of optical properties of $\mathbf{G a}(\mathbf{I n})$ AsBi quantum well systems using 8band and 14-band models [9742-45]

iv 
974219 Influence of p-doping on the gain and refractive index dynamics in quantum dash based semiconductor optical amplifiers [9742-46]

SESSION 11 PHOTOVOLTAICS MODELING: JOINT SESSION WITH CONFERENCES 9742 AND 9743

97421 A Theoretical insights into hybrid perovskites for photovoltaic applications (Invited Paper) [9742-47]

9742 1B Theoretical studies of Rashba and Dresselhaus effects in hybrid organic-inorganic perovskites for optoelectronic applications sample [9742-48]

\section{SESSION 12 PLASMONICS}

$97421 \mathrm{E}$ Transmittance of long-wavelength infrared surface plasmon by hexagonal periodic metal hole arrays [9742-52]

9742 IG Nonlinear scattering in gold nanospheres [9742-54]

SESSION 13 RESONATORS AND GRATINGS

$974211 \quad$ Mid-IR high-index dielectric Huygens metasurfaces [9742-56]

$97421 \mathrm{~J}$ Enhancement of effective quality-factor using asymmetric Mach-Zehnder interferometer with ring resonator for optical bio and chemical sensor [9742-57]

\section{SESSION 14 NUMERICAL METHODS AND PROCESS SIMULATION}

9742 IL Efficient and accurate modelling of quantum nanostructures [9742-59]

$97421 \mathrm{M}$ Enhancing accuracy with subpixel smoothing for multiband effective-mass Hamiltonians of semiconductor nanostructures [9742-60]

$974210 \quad$ Numerical analysis using 2D modeling of optical fiber poled by induction [9742-62]

POSTER SESSION

9742 1Q Absorption enhancement by textured InP in solar cells [9742-49]

9742 IR Industrial robot's vision systems [9742-63]

$97421 \mathrm{~T}$ The simulation and experimental research on the sensing characteristics of few-mode-fiber based LPFG [9742-65]

$97421 \mathrm{~V}$ Characterization of the non-collinear acousto-optical cell based on calomel $\left(\mathrm{Hg}_{2} \mathrm{Cl}_{2}\right)$ crystal and operating within the two-phonon light scattering [9742-67] 
$97421 Y$ Dielectric elastomer-based laser beam pointing method with ultraviolet and visible wavelength [9742-72]

974221 Development of new maskless manufacturing method for anti-reflection structure and application to large-area lens with curved surface [9742-75]

974222 III-nitride monolithic LED covering full RGB color gamut [9742-77] 


\title{
Authors
}

Numbers in the index correspond to the last two digits of the six-digit citation identifier (CID) article numbering system used in Proceedings of SPIE. The first four digits reflect the volume number. Base 36 numbering is employed for the last two digits and indicates the order of articles within the volume. Numbers start with 00, 01, 02, 03, 04, 05, 06, 07, 08, 09, OA, OB...0Z, followed by 10-1Z, 20-2Z, etc.

\author{
Arellanes, Adan Omar, IV \\ Argyris, Apostolos, OL \\ Ayad, Marina, $1 \mathrm{~L}$ \\ Babazadeh, N., OS \\ Bellotti, Enrico, 02 \\ Bertazzi, Francesco, 02 \\ Bourmpos, Michail, OL \\ Boyer-Richard, Soline, 1 A \\ Burger, Sven, OM \\ Burte, Edmund P., 08 \\ Butler, Thomas, OK \\ Calciati, Marco, 02 \\ Carignano, Marcelo, $1 \mathrm{~A}$ \\ Carmele, Alexander, 0X, 16 \\ Chan, Sze-Chun, OC \\ Chang, Chi-Hsiang, 14 \\ Chang, Shu-Wei, ON, 14, 1M \\ Chen, Guanghui, IT \\ Cheng, Pi-Ju, ON \\ Childs, D. T., OS \\ Chimot, Nicolas, 19 \\ Choi, Young-Wan, 1J \\ Chu, Shi-Wei, $1 \mathrm{G}$ \\ Chuang, Chih-Li, 22 \\ Clavel, Michael B., 11 \\ Corbari, C., 10 \\ Cui, Kaiyu, $\mathrm{OZ}$ \\ De Lucia, F., 10 \\ De Santi, Carlo, 02 \\ Debernardi, Pierluigi, 02 \\ Ding, Jun, 11 \\ Dolcini, Fabrizio, 02 \\ Dominici, Stefano, 02 \\ Droenner, Leon, 16 \\ Dubinkin, llya, OD \\ El-Ghoroury, Hussein S., 22 \\ Erneux, Thomas, OD \\ Even, Jacky, 1A, 1B \\ Fedorov, Nikita, OD \\ Fedosovsky, Michael E, IR \\ Feng, Xue, $O Z$ \\ Fu, Y., OA \\ Fukui, Hidetoshi, 21 \\ Ghione, Giovanni, 02 \\ Gladysiewicz, M., 18 \\ Goano, Michele, 02 \\ Goulding, David, OD, OK \\ Grillot, F., OE, OG \\ Hader, J., $\mathrm{OH}$
}

\author{
Hammerschmidt, Martin, OM \\ Haruta, Yasuyuki, 21 \\ Hayakawa, Tomohiko, 1 Y \\ Häyrynen, Teppo, 17 \\ Healy, N., 10 \\ Hegarty, Stephen P., OD, OK \\ Hein, Sven M., OX, 16 \\ Herrmann, Sven, OM \\ Hogg, R. A., OS \\ Houlihan, John, 19 \\ Hsieh, Chi-Ti, 1M \\ Hsieh, Tung-Han, IM \\ Hu, Juejun, 11 \\ Huang, D., 10 \\ Huang, Yidong, $\mathrm{OZ}$ \\ Hudait, Mantu K., 11 \\ Huyet, Guillaume, OD, OK, 19 \\ Ishikawa, Masatoshi, IY \\ lureva, Radda A., IR \\ Ivashev, I., 18 \\ Jancu, Jean-Marc, $1 \mathrm{~A}$ \\ Ji, Myung-Gi, $1 \mathrm{~J}$ \\ Ji, Taeksoo, 1Q \\ Joshi, Siddharth, 19 \\ Jung, Mi, $1 \mathrm{~J}$ \\ Kabuss, Julia, 16 \\ Karnowski, Karol, OK \\ Karpiak, Bogdan, 07 \\ Katan, Claudine, 1A, 1B \\ Kelleher, Bryan, OD, OK \\ Kepenekian, M., 1B \\ Kilen, I., $\mathrm{OH}$ \\ Kim, Doo Gun, 1 J \\ Kim, Ha Sul, 1E \\ Kim, Hong-Seung, 1J \\ Kim, Tae-Ryong, 1 J \\ Kisin, Mikhail V., 22 \\ Kitagawa, Seiichiro, 21 \\ Knorr, Andreas, 0X, 16 \\ Koch, S. W., $\mathrm{OH}$ \\ Komarov, Igor I., IR \\ Komolibus, Katarzyna, 19 \\ Kovalev, Anton V., OP \\ Kwak, Hoe Min, $1 E$ \\ LaRochelle, S., OE, OG \\ Lee, Byungwoo, $1 \mathrm{E}$ \\ Lee, Tae-Kyeong, $1 \mathrm{~J}$ \\ Lelarge, Francois, 19 \\ Lestrade, M., OA
}


Li, Song-Sui, OC

Li, Xiao-Zhou, OC

Li, Yongzhuo, OZ

Li, Z. M. Simon, OA

Li, Z. Q., OA

Lin, Cheng-Wei, $1 G$

Lin, Yuankun, 11

Liu, Hsiang-Lin, $1 G$

Lyu, Hong Chou, OK

Maltseva, Nadezhda K., IR

Mandurrino, Marco, 02

Meneghini, Matteo, 02

Moloney, J. $\mathrm{V} ., \mathrm{OH}$

Morohashi, Isao, OJ

Murphy-Armando, Felipe, 11

Naumann, Nicolas L., 16

Nishi, K., OS

O'Shaughnessy, Ben, OK

Obaya, Salah S. A., 1L

Ochalski, Tomasz J., 11

Oh, Guem-Yoon, 1J

Oksanen, Jani, 17

Partanen, Mikko, 17

Pedesseau, Laurent, 1A, 1B

Pikhtin, Nikita, Ol

Piwonski, Tomasz, 19

Podoskin, Alexsandr, 0

Polyakov, Vadim M., OP

Pomplun, Jan, OM

Puers, Robert, 07

Raskin, Evgeni O., IR

Ren, Han, 11

Resetar, Tomislav, 07

Robles, R., 1B

Rossi, Fausto, 02

Sabri Alirezaei, Iman, 08

Sakamoto, Takahide, 0J

Saladukha, Dzianis, 11

Sang, Mei, IT

Sapori, D., 1B

Sarraute, J.-M., OE, OG

Sattar, Zubaida A., OB

Sazio, P. J. A., 10

Schires, K., OE, OG

Schmidt, Frank, OM

Shcherbakov, Alexandre S., IV

Shen, Po-Ting, $1 \mathrm{G}$

Shore, K. Alan, OB

Slepneva, Svetlana, OK

Slipchenko, Sergey, 이

Sobhani, S. A., OS

Soboleva, Olga, ol

Son, Byung-Hee, $1 \mathrm{~J}$

Stevens, B. J., OS

Sugawara, M., OS

Süss, Andreas, 07

Swillam, Mohamed A., 1L

Syvridis, Dimitris, OL

Takaoka, Toshimitsu, 21

Takemasa, K., OS
Tarasov, Il'ya, ol

Tibaldi, Alberto, 02

Trivellin, Nicola, 02

Tulkki, Jukka, 17

Tykalewicz, Bogsulaw, OD

Uehara, K., OA

Vallone, Marco, 02

Van Hoof, Chris, 07

Vermandere, Elke, 07

Verzellesi, Giovanni, 02

Viktorov, Evgeny A., OD

Vladimirov, Andrei G., OK

Wang, Biao, $1 T$

Wang, Lihui, $1 Y$

Wang, $Y u, O Z$

Wartak, M. S., 18

Wojtkowski, Maciej, OK

Wu, Chao-Hsin, 14

Xiao, Y. G., OA

Yamamoto, Kazuya, 21

Yamashita, Tomoya, 21

Yuferev, Valentin, 0 I

Yun, Seokhun, 1Q

Zanoni, Enrico, 02

Zhang, Hualiang, 11

Zhang, Li, 1 I

Zhang, Weigang, IT

Zhong, Chenhao, $1 \mathrm{~T}$

Zhou, Mi, 1 I

Zhou, Xiangyu, 02

Zhou, Y. J., OA

Zhuang, Jun-Ping, OC 


\section{Conference Committee}

Symposium Chairs

Jean-Emmanuel Broquin, IMEP-LAHC (France)

Shibin Jiang, AdValue Photonics, Inc. (United States)

Symposium Co-Chairs

David L. Andrews, University of East Anglia (United Kingdom)

Alexei L. Glebov, OptiGrate Corporation (United States)

Program Track Chair

James G. Grote, Air Force Research Laboratory (United States)

Conference Chairs

Bernd Witzigmann, Universität Kassel (Germany)

Marek Osiński, The University of New Mexico (United States)

Yasuhiko Arakawa, The University of Tokyo (Japan)

Conference Program Committee

Hiroshi Amano, Nagoya University (Japan)

Toshihiko Baba, Yokohama National University (Japan)

Enrico Bellotti, Boston University (United States)

Guillermo Carpintero del Barrio, Universidad Carlos III de Madrid (Spain)

Weng W. Chow, Sandia National Laboratories (United States)

Alexandre Freundich, University of Houston (United States)

Frédéric Grillot, Télécom ParisTech (France)

Ortwin Hess, Imperial College London (United Kingdom)

Thomas A. Klar, Johannes Kepler Universität Linz (Austria)

Stephan W. Koch, Philipps-Universität Marburg (Germany)

Cun-Zheng Ning, Arizona State University (United States)

Joachim Piprek, NUSOD Institute LLC (United States)

Marc Sciamanna, Supélec (France)

Ikuo Suemune, Hokkaido University (Japan) 


\section{Session Chairs}

$1 \quad$ Light Emitting Diodes

Sze-Chun Chan, City University of Hong Kong (Hong Kong, China)

2 Photodetectors

K. Alan Shore, Bangor University (United Kingdom)

3 Optical Injection and Feedback

Frédéric Grillot, Télécom ParisTech (France)

4 Nonlinear Laser Dynamics

Marek Osiński, The University of New Mexico (United States)

5 Electromagnetics

Bernd Witzigmann, Universität Kassel (Germany)

6 Quantum Dots

Jacky Even, Institut National des Sciences Appliquées de Rennes (France)

7 Non-Classical Light

Christopher Gies, Universität Bremen (Germany)

8 Silicon Opto-/Electronics

Weng W. Chow, Sandia National Labs (United States)

9 Semiconductor Lasers

Cun-Zheng Ning, Arizona State University (United States)

10 Active Materials

Sergio Nicoletti, MINATEC (France)

11 Photovoltaics Modeling: Joint Session with Conferences 9742 and 9743

Alexandre Freundlich, University of Houston (United States)

Bernd Witzigmann, Universität Kassel (Germany)

12 Plasmonics

Giovanni Capellini, IHP GmbH (Germany)

13 Resonators and Gratings

Thomas A. Klar, Johannes Kepler Universität Linz (Austria)

14 Numerical Methods and Process Simulation

Yidong Huang, Tsinghua University (China) 